\title{
EVOLUTION GENERATED BY SEMILINEAR DISSIPATIVE PLUS COMPACT OPERATORS
}

\author{
BY \\ ERIC SCHECHTER ${ }^{1}$
}

\begin{abstract}
Existence results and sharp continuous dependence results are given for an evolution equation in an arbitrary Banach space. The right-hand side of the equation consists of a linear dissipative term plus a continuous dissipative term plus a compact term.
\end{abstract}

1. Introduction. In this paper we consider problems of the form

$$
u^{\prime}(t)=D(u(t))+f(t)
$$

and

$$
u^{\prime}(t)=D(u(t))+C(t, u(t))
$$

Here $D$ is a semilinear (i.e., continuous plus linear) $m$-dissipative operator in a Banach space; $f$ takes values in a compact set; and $C$ is a compact operator. Many partial differential equations can be written in the form $u^{\prime}(t)=D(u(t))$; see $[4,13]$ for examples. The compact perturbation in (1.2) enables us to consider differentialintegral equations; see [13] for examples of integral operators in evolution equations.

After some preliminary definitions and lemmas, in $\$ 4$ we consider the dependence of $u$ on $f$ in (1.1). It is well known that if $D$ is $m$-dissipative and $u(0) \in \operatorname{cl}(\operatorname{Dom}(D))$ is fixed, then the mapping $f \mapsto u$ determined by (1.1) is a nonexpansive mapping from $\mathcal{L}^{1}([0, T], X)$ into $\mathcal{C}([0, T], X)$. (This result is reviewed in Proposition 1 in $\S 2$.) In $\S 4$ we obtain a continuous dependence result which is sharper in some respects. The mapping $f \mapsto u$ is continuous from a topology substantially weaker than that of $\mathcal{L}^{1}([0, T], X)$, into $\mathcal{C}([0, T], X)$, provided that $D$ is semilinear and the functions $f$ being considered all have ranges contained in some fixed compact subset of $X$. The proof is a variant of techniques in [16]. We also give a converse, which shows that the topology cannot be weakened further; and an example, which shows that the compactness assumption cannot be omitted.

The retopologized space $\mathcal{L}^{\prime}([0, T], X)$, having a weaker topology, has more compact subsets. Thus it is more amenable to compactness arguments, such as the Schauder-Tychonoff Fixed Point Theorem. In $\$ 5$ we apply that theorem, together

Received by the editors November 16, 1981 and, in revised form, December 21, 1981.

1980 Mathematics Subject Classification. Primary 34G20; Secondary 34A10, 47H20.

Key words and phrases. Accretive, compact, continuous dependence, dissipative, evolution, perturbation, semilinear.

'Supported in part by a grant from the Vanderbilt University Research Council.

(C) 1983 American Mathematical Society 0002-9947/81/0000-0756/\$04.00 
with our continuous dependence results, to prove existence of solutions to (1.2). This result extends in part the results in [17 and 19].

The argument in $\$ 3$ of this paper uses the continuity of the nonlinear part of $D$. We remark that this continuity hypothesis can be weakened slightly. For instance, if $D$ is a partial differential operator (not necessarily linear) with fairly smooth coefficients, then $D$ may be " $\nu$-continuous" in the sense of [18]. In such cases, $D$ behaves much like a continuous operator. Hence the methods of the present paper are applicable, although in a much more complicated form.

The argument in $\$ 3$ also requires that $D$ be densely defined in $X$. An example in $\$ 4$ shows that our continuous dependence result may fail if $D$ is linear and $m$-dissipative but not densely defined. For such operators, or for operators which are not semilinear and do not satisfy a $\nu$-continuity condition, it is not yet known whether an existence theorem analogous to that in $\$ 5$ can be obtained by some other method. Thus the following question is still open: Let $D$ be an arbitrary $m$-dissipative operator in $X$, and let $C$ be compact. Does (1.2) necessarily have a solution? This certainly is plausible, since each of the two simpler equations

$$
\begin{gathered}
u^{\prime}(t)=D(u(t))+f(t), \\
u^{\prime}(t)=C(t, u(t))+f(t)
\end{gathered}
$$

has a solution. But the existence theory for (1.3) is based on very delicate metric estimates $[7,8]$, whereas the theory for $(1.4)$ is topological and largely nonmetric. There is still a gap between these two theories.

The author is grateful to Bob Martin and Glenn Webb for their advice and encouragement.

2. Preliminaries. Most of the results in this section can be found in Barbu [3] or Goldstein [12]; we shall follow the notations of those sources. Many of these results can also be found in $[5,6,7,9,13]$, with various notations.

Throughout this paper we assume $(X,\|\|)$ is a Banach space. Define $\langle,\rangle_{+}$: $X \times X \rightarrow R$ by

$$
\langle x, y\rangle_{+}=\lim _{\lambda \downarrow 0}(\|x+\lambda y\|-\|x\|) / \lambda=\inf _{\lambda>0}(\|x+\lambda y\|-\|x\|) / \lambda
$$

the last equality follows from the fact that $\|x+\lambda y\|$ is a convex function of $\lambda$. Note that $\left|\langle x, y\rangle_{+}\right| \leqslant\|y\|$; and that $\langle,\rangle_{+}$is jointly upper semicontinuous since it is the infimum of a family of continuous functions.

Let Dom, Ran, cl, dist denote domain, range, closure, and distance, respectively. Let $A$ be a mapping from some set $\operatorname{Dom}(A) \subseteq X$ into $X$. $A$ is dissipative (equivalently, $-A$ is accretive ) if $\left\langle x_{1}-x_{2},-A\left(x_{1}\right)+A\left(x_{2}\right)\right\rangle_{+} \geqslant 0$ for all $x_{1}, x_{2}$ in $\operatorname{Dom}(A)$. Equivalently, $A$ is dissipative if for each $\lambda>0, I-\lambda A$ is injective and its inverse is Lipschitz with $\left\|(I-\lambda A)^{-1}\right\|_{\text {Lip }} \leqslant 1$. Other equivalent definitions can also be found in the literature; see especially $[3,6,12,13]$. We remark that most of the references consider multivalued operators $A$; but such greater generality is not useful in the present paper. Also, many of the references consider the more general case of operators $A$ such that $A-\omega I$ is dissipative for some constant $\omega$; but for simplicity we shall take $\omega=0$. 
$A$ is $m$-dissipative if it is dissipative and $\operatorname{Ran}(I-\lambda A)=X$ for some $\lambda>0$. It then follows [3, Proposition II.3.3] that $\operatorname{Ran}(I-\lambda A)=X$ for every $\lambda>0$.

For any interval $J \subseteq R$, let $\mathcal{L}^{1}(J, X)$ and $\mathcal{C}(J, X)$ be the Banach spaces of integrable functions and bounded continuous functions from $J$ into $X$, respectively, with their usual norms \|\|$_{1}$ and \|\|$_{\infty}$. Let $\varrho_{\text {loc }}^{1}(R, X)$ be the vector space of locally integrable functions, i.e., functions integrable on bounded intervals in $R$. lloc $_{\text {loc }}(R, X)$ is usually topologized as a Fréchet space, with $f_{n} \rightarrow f_{\infty}$ in $\rho_{\text {loc }}^{1}(R, X)$ if

$$
\int_{a}^{b}\left\|f_{n}(t)-f_{\infty}(t)\right\| d t \rightarrow 0 \text { for all } a, b .
$$

We consider the quasiautonomous differential equation

$$
u^{\prime}(t)=A(u(t))+f(t) \quad(t \in J)
$$

for $A$ m-dissipative and $f \in \mathcal{L}^{\mathrm{l}}(J, X)$. An integral solution of $(2.1)$ is a continuous function $u: J \rightarrow \operatorname{cl}(D(A))$ such that

$$
\|u(t)-x\|-\|u(r)-x\| \leqslant \int_{r}^{t}\langle u(s)-x, f(s)+A(x)\rangle_{+} d s
$$

whenever $r \leqslant t$ in $J$ and $x \in \operatorname{Dom}(A)$. We emphasize that $u$ is not necessarily differentiable; equation (2.1) is used as a symbolic shorthand for (2.2). The notion of integral solutions extends more classical notions of differentiable solutions; see $[5,6,11,12]$ for examples of the usefulness of this extension.

We now briefly review some properties of $m$-dissipative operators and integral solutions which will be needed later in this paper.

Proposition 1. Let $A$ be an m-dissipative operator in $X$. Then for each $x \in$ $\operatorname{cl}(\operatorname{Dom}(A)), f \in \mathcal{L}_{\mathrm{loc}}^{\mathrm{l}}(R, X)$ and $a \in R$, there exists an integral solution of $u^{\prime}(t)=$ $A(u(t))+f(t)(t \geqslant a), u(a)=x$. The solution $u(t)$ is uniquely determined by $t, f, a, x$; the mapping $u(t)=u(t, f, a, x)$ is jointly continuous from $\{(t, f, a, x) \in R \times$ $\left.\mathrm{E}_{\text {loc }}^{1}(R, X) \times R \times X: t \geqslant a ; x \in \operatorname{cl}(\operatorname{Dom}(A))\right\}$ into $X$. For fixed $a$, we have the following estimate: if $u_{1}$ and $u_{2}$ are the integral solutions corresponding to data $\left(x_{1}, f_{1}\right)$ and $\left(x_{2}, f_{2}\right)$, then

$$
\begin{aligned}
\left\|u_{1}(t)-u_{2}(t)\right\| & \leqslant\left\|x_{1}-x_{2}\right\|+\int_{a}^{t}\left\langle u_{1}(s)-u_{2}(s), f_{1}(s)-f_{2}(s)\right\rangle_{+} d s \\
& \leqslant\left\|x_{1}-x_{2}\right\|+\int_{a}^{t}\left\|f_{1}(s)-f_{2}(s)\right\| d s, \text { for all } t \geqslant a .
\end{aligned}
$$

Proof. Most of the above result, including (2.3), can be found in $[5,6,7,12]$. To prove joint continuity, note that $u\left(t_{1}-h, f, a, x\right)=u\left(t_{1}, g, a+h, x\right)$ where $g(s)$ $=f(s-h)$. Let $t_{1} \geqslant a+h$ and $t \geqslant a$. By (2.3),

$$
\begin{aligned}
\| u\left(t_{1}, f_{1}, a+h, x_{1}\right) & -u(t, f, a, x)\|\leqslant\| u\left(t_{1}, f_{1}, a+h, x_{1}\right)-u\left(t_{1}, g, a+h, x\right) \| \\
& +\left\|u\left(t_{1}-h, f, a, x\right)-u(t, f, a, x)\right\| \\
\leqslant & \left\|x_{1}-x\right\|+\int_{a+h}^{t_{1}}\left[\left\|f_{1}(s)-f(s)\right\|+\|f(s)-f(s-h)\|\right] d s \\
& +\left\|u\left(t_{1}-h, f, a, x\right)-u(t, f, a, x)\right\|
\end{aligned}
$$

which tends to 0 when $t_{1} \rightarrow t, f_{1} \rightarrow f, h \rightarrow 0$, and $x_{1} \rightarrow x$. 
REMARK. Our main result, in $\S 4$ of this paper, is in some respects a sharpening of (2.3); see Remark 2 in $\S 4$.

Proposition 2. Let $A$ be an m-dissipative operator in $X$. Let $B: X \rightarrow X$ be continuous, everywhere defined, and dissipative. Then:

(i) $B$ is totally dissipative. That is, $\left\langle x_{1}-x_{2}, B\left(x_{1}\right)-B\left(x_{2}\right)\right\rangle_{+} \leqslant 0$ for all $x_{1}, x_{2}$ $\in X$.

(ii) $B$ is m-dissipative.

(iii) $A+B$ is $m$-dissipative (with $\operatorname{Dom}(A+B)=\operatorname{Dom}(A)$ ).

(iv) Let $u_{0} \in \operatorname{cl}(\operatorname{Dom}(A))$ and $f \in \mathcal{L}^{\mathrm{l}}([0, T], X)$. Then there exist $g \in \mathcal{L}^{\mathrm{l}}([0, T], X)$ and $u \in \mathcal{C}([0, T], X)$ such that $g(t)=B(u(t))+f(t)$ and $u$ is an integral solution of $u^{\prime}(t)=A(u(t))+g(t)(0 \leqslant t \leqslant T), u(0)=u_{0}$.

(v) Suppose $u_{0}, f, g, u$ satisfy the hypotheses and conclusions of part (iv), above. Then $u$ is the integral solution of $u^{\prime}(t)=(A+B)(u(t))+f(t)(0 \leqslant t \leqslant T), u(0)=u_{0}$.

Proof. Part (i) follows from Proposition I.1.1 and Lemma III.3.2 in [3]. For later reference we note that (i) implies

$$
\begin{aligned}
\langle u-x, B(u)+v\rangle_{+} & \leqslant\langle u-x, B(u)-B(x)\rangle_{+}+\langle u-x, B(x)+v\rangle_{+} \\
& \leqslant\langle u-x, B(x)+v\rangle_{+}
\end{aligned}
$$

for any $u, v, x \in X$.

Part (ii) is a special case of (iii), since we can take $A=0$. Part (iii) can be found in [14] (see Remarque $\left(2^{\circ}\right)$ ), or in [2].

We shall prove part (v) before part (iv). Suppose $u_{0}, f, g, u$ satisfy the hypotheses and the conclusions of part (iv). Then for any $x \in \operatorname{Dom}(A)$ and $0 \leqslant r \leqslant t \leqslant T$,

$$
\begin{aligned}
\|u(t)-x\|-\|u(r)-x\| & \leqslant \int_{r}^{t}\langle u(s)-x, g(s)+A(x)\rangle_{+} d s \\
& =\int_{r}^{t}\langle u(s)-x, B(u(s))+f(s)+A(x)\rangle_{+} d s \\
& \leqslant \int_{r}^{t}\langle u(s)-x, B(x)+f(s)+A(x)\rangle_{+} d s ;
\end{aligned}
$$

the last inequality follows from (2.4). This proves part (v).

For (iv), let any $u_{0} \in \operatorname{cl}(\operatorname{Dom}(A))$ and $f \in \mathcal{L}^{1}([0, T], X)$ be given. Choose some sequence of continuous functions $f_{n}$ which converge in $\mathcal{L}^{1}([0, T], X)$ to $f$. Passing to a subsequence, we may assume that $f_{n}(t) \rightarrow f(t)$ for almost all $t$, and that the sequence $\left\{f_{n}\right\}$ is dominated by a single function in $\mathcal{L}^{\mathrm{l}}([0, T], R)$.

For each $n$, the function $B_{n}(t, x) \equiv B(x)+f_{n}(t)$ is jointly continuous, and totally dissipative in $x$. By Theorem III of [14], there exist functions $g_{n}, u_{n} \in \mathcal{C}([0, T], X)$ such that $g_{n}(t)=B_{n}\left(t, u_{n}(t)\right)=B\left(u_{n}(t)\right)+f_{n}(t)$ and $u_{n}$ is an integral solution of $u_{n}^{\prime}(t)=A\left(u_{n}(t)\right)+g_{n}(t)(0 \leqslant t \leqslant T), u_{n}(0)=u_{0}$. That is,

$$
\left\|u_{n}(t)-x\right\|-\left\|u_{n}(r)-x\right\| \leqslant \int_{r}^{t}\left\langle u_{n}(s)-x, g_{n}(s)+A(x)\right\rangle_{+} d s
$$

whenever $x \in \operatorname{Dom}(A)$ and $0 \leqslant r \leqslant t \leqslant T$. By part (v) above, $u_{n}$ is an integral solution of $u_{n}^{\prime}(t)=(A+B)\left(u_{n}(t)\right)+f_{n}(t)(0 \leqslant t \leqslant T), u_{n}(0)=u_{0}$. 
By Proposition 1 above, $\left\|u_{m}-u_{n}\right\|_{\infty} \leqslant\left\|f_{m}-f_{n}\right\|_{1}$. Hence the functions $u_{n}$ converge uniformly on $[0, T]$ to some $u \in \mathcal{C}([0, T], X)$; hence $B\left(u_{n}(\cdot)\right) \rightarrow B(u(\cdot))$ uniformly also. Then $g_{n}(t) \rightarrow g(t) \equiv B(u(t))+f(t)$, for almost all $t$; and the convergence is dominated by some function $h \in \mathcal{L}^{1}([0, T], R)$. For fixed $s$,

$$
\limsup _{n \rightarrow \infty}\left\langle u_{n}(s)-x, g_{n}(s)+A(x)\right\rangle_{+} \leqslant\langle u(s)-x, g(s)+A(x)\rangle_{+},
$$

since $\langle,\rangle_{+}$is upper semicontinuous. Also

$$
\left|\left\langle u_{n}(s)-x, g_{n}(s)+A(x)\right\rangle_{+}\right| \leqslant\left\|g_{n}(s)+A(x)\right\| \leqslant h(s)+\|A(x)\| .
$$

Take limits in (2.5); using the dominated version of Fatou's lemma, we obtain

$$
\|u(t)-x\|-\|u(r)-x\| \leqslant \int_{r}^{t}\langle u(s)-x, g(s)+A(x)\rangle_{+} d s .
$$

This completes the proof of Proposition 2.

We shall also need some properties of the autonomous differential equation

$$
u^{\prime}(t)=A(u(t)) \quad(t \geqslant 0) .
$$

Assume $A$ is $m$-dissipative. It follows from Proposition 1 (with $f \equiv 0$ ) that the integral solutions of (2.6) are given by $u(t)=\phi(t, u(0))$, for some jointly continuous function $\phi=\phi_{A}:[0, \infty) \times \operatorname{cl}(\operatorname{Dom}(A)) \rightarrow \operatorname{cl}(\operatorname{Dom}(A))$. This function $\phi$ also satisfies the semigroup properties: $\phi(0, x)=x, \phi(t, \phi(s, x))=\phi(t+s, x)$ for all $x \in$ $\operatorname{cl}(\operatorname{Dom}(A))$ and $t, s \geqslant 0$; and the contraction property: $\|\phi(t, x)-\phi(t, y)\| \leqslant \| x-$ $y \|$ for all $x, y \in \operatorname{cl}(\operatorname{Dom}(A))$ and $t \geqslant 0$. We often write $\phi(t, x)=e^{t A} x=\exp (t A) x$, because $\phi(t, x)=\lim _{n \rightarrow \infty}(I-t A / n)^{-n} x[3,8]$.

For the purposes of this paper, our main interest in $\exp (t A)$ is for $A$ linear and densely defined. For such $A, \exp (t A)$ is linear, and $\|\exp (t A) x\| \leqslant\|x\|$.

Proposition 3. Let $A$ be a linear, $m$-dissipative operator in $X$, with $\operatorname{Dom}(A)$ dense in $X$. Let $g \in \mathcal{L}^{\mathrm{l}}([0, T], X)$ and $u_{0} \in X$. Then the integral solution of $u^{\prime}(t)=A(u(t))$ $+g(t)(0 \leqslant t \leqslant T), u(0)=u_{0}$, is given by

$$
u(t)=\exp (t A) u_{0}+\int_{0}^{t} \exp ((t-s) A) g(s) d s \quad(0 \leqslant t \leqslant T) .
$$

Proof. If $g$ is continuously differentiable and $u_{0} \in \operatorname{Dom}(A)$, then [3, Proposition I.3.2] the function $u$ defined by (2.7) is continuously differentiable, and satisfies the differential equation $u^{\prime}(t)=A(u(t))+g(t)(0 \leqslant t \leqslant T)$. It then follows easily that $u$ is also an integral solution of this differential equation. For general $g \in$ $\mathcal{L}^{1}([0, T], X)$ and $u_{0} \in \operatorname{cl}(\operatorname{Dom}(A))$, take limits using (2.3); we omit the details.

Corollary. Let $A$ be a linear, densely defined, $m$-dissipative operator in $X$. Let $B$ : $X \rightarrow X$ be continuous, everywhere defined, and dissipative. Let $f \in \mathcal{L}_{\mathrm{loc}}^{1}(R, X), a \in$ $R, x \in X$. Then the following three conditions are equivalent, and there is a unique function $u$ satisfying them:

(i) $u$ is the integral solution of $u^{\prime}(t)=(A+B)(u(t))+f(t)(t \geqslant a), u(a)=x$.

(ii) $u$ is the integral solution of $u^{\prime}(t)=A(u(t))+g(t)(t \geqslant a), u(a)=x$, for some function $g \in \mathrm{L}_{\text {loc }}^{1}(R, X)$; and $g(t)=B(u(t))+f(t)$. 
(iii) $u$ is a continuous map from $[a, \infty)$ into $X$, and

$$
u(t)=\exp ((t-a) A) x+\int_{a}^{t} \exp ((t-s) A)[B(u(s))+f(s)] d s
$$

for all $t \geqslant a$.

The remainder of this paper is concerned with the function $u(t)$ described in the corollary above. We shall hold the operators $A$ and $B$ fixed, and consider the dependence of $u$ on varying $t, f, a, x$. We write $u(t)=u(t, f, a, x)$ to display this dependence.

3. Technical lemma. For notation see the end of the previous section.

Lemma. Let $A$ be a linear, densely defined, $m$-dissipative operator in $X$. Let $B$ : $X \rightarrow X$ be continuous, everywhere defined, and dissipative. Let $K$ be a compact subset of $X$, and let $\varepsilon>0$. Then there exists $\delta=\delta(\varepsilon, K)>0$ with the following properties:

Suppose $0<h \leqslant \delta, a \in R, x \in K$, and $f, g \in \mathcal{L}_{\text {loc }}(R, X)$ with $\operatorname{Ran}(f) \cup \operatorname{Ran}(g)$ $\subseteq K$. Then

$$
\|u(a+h, f, a, x)-x\|<\varepsilon,
$$

and

$$
\left\|u(a+h, f, a, x)-u(a+h, g, a, x)-\int_{a}^{a+h}[f(s)-g(s)] d s\right\|<h \varepsilon .
$$

Proof. Let $\alpha=\max \{\|x\|: x \in K\}$. Since $B$ is continuous and $K$ is compact,

$$
\beta \equiv \sup \{\|B(y)\|: y \in X ; \operatorname{dist}(y, K) \leqslant \rho\}
$$

is finite for $\rho>0$ sufficiently small. Fix any such $\rho$ and $\beta$. Let

$$
\begin{aligned}
& \gamma_{1}(h)=\sup \{\|B(x)-B(y)\|: x \in K, y \in X,\|x-y\| \leqslant h\}, \\
& \gamma_{2}(h)=\max \{\|\exp (t A) x-x\|: x \in K, t \in[0, h]\} .
\end{aligned}
$$

Then $\gamma_{1}(h)$ and $\gamma_{2}(h)$ decrease to 0 when $h \downarrow 0$ since $K$ is compact, $B$ is continuous, and $(t, x) \mapsto \exp (t A) x$ is jointly continuous.

Note that $\gamma_{2}(h)+(\beta+\alpha) h<\rho$ for all $h \geqslant 0$ sufficiently small. We claim that

$$
\begin{aligned}
\|u(a+h, f, a, x)-x\| \leqslant \gamma_{2}(h)+ & (\beta+\alpha) h \\
& \text { whenever } \gamma_{2}(h)+(\beta+\alpha) h<\rho,
\end{aligned}
$$

for $x \in K$ and $\operatorname{Ran}(f) \subseteq K$. To see this, for brevity let $u(t)=u(t, f, a, x)$. Since $u(a)=x$ and $u$ is continuous, $\|u(t)-x\|<\rho$ for all $t$ in some interval $[a, T)$. Choose $T$ as large as possible; it may be $\infty$. Note that if $T<\infty$ then $\|u(T)-x\|=$ $\rho$.

By (2.8), if $t \in[a, \infty)$ and $t \leqslant T$, then

$$
\begin{aligned}
\|u(t)-x\| & \leqslant\|\exp ((t-a) A) x-x\|+\int_{a}^{t}\{\|B(u(s))\|+\|f(s)\|\} d s \\
& \leqslant \gamma_{2}(t-a)+(\beta+\alpha)(t-a)
\end{aligned}
$$


In particular, if $T<\infty$, (3.4) yields

$$
\rho=\|u(T)-x\| \leqslant \gamma_{2}(T-a)+(\beta+\alpha)(T-a) .
$$

The right side of (3.4) is a nondecreasing function of $t$. Hence $\gamma_{2}(h)+(\beta+\alpha) h<\rho$ implies $a+h<T$, whether $T$ is finite or not. This proves (3.3).

Choose some $\lambda>0$ small enough so that $\gamma_{1}(\lambda)<\varepsilon / 4$. Then choose some $\delta>0$ small enough so that $\gamma_{2}(\delta)+(\beta+\alpha) \delta<\min \{\varepsilon / 4, \rho, \lambda\}$. We shall show that this choice of $\delta$ satisfies the requirements of the lemma. Inequality (3.1) follows from (3.3); it suffices to verify (3.2).

For brevity let $f_{1}=f$ and $f_{2}=g$. For $j=1,2$, let $u_{j}(t)=u\left(t, a, f_{j}, x\right)$. For $a \leqslant s \leqslant a+h \leqslant a+\delta$, by (3.3) we have $\left\|u_{j}(s)-x\right\|<\lambda$; hence

$$
\left\|B\left(u_{j}(s)\right)-B(x)\right\| \leqslant \gamma_{1}(\lambda)<\varepsilon / 4
$$

hence $\left\|B\left(u_{1}(s)\right)-B\left(u_{2}(s)\right)\right\|<\varepsilon / 2$. Also $\left\|\exp ((a+h-s) A) f_{j}(s)-f_{j}(s)\right\| \leqslant$ $\gamma_{2}(h)<\varepsilon / 4$. By $(2.8)$,

$$
\begin{aligned}
& \left\|u_{1}(a+h)-u_{2}(a+h)-\int_{a}^{a+h}\left[f_{1}(s)-f_{2}(s)\right] d s\right\| \\
& \leqslant \int_{a}^{a+h}\left\|B\left(u_{1}(s)\right)-B\left(u_{2}(s)\right)\right\| d s \\
& \quad+\sum_{j=1}^{2} \int_{a}^{a+h}\left\|\exp ((a+h-s) A) f_{j}(s)-f_{j}(s)\right\| d s
\end{aligned}
$$

which is less than $\varepsilon h$. This completes the proof of the lemma.

4. Continuous dependence. For notation see the end of $\S 2$.

Theorem 1. Let $A$ be a linear, densely defined, $m$-dissipative operator in $X$. Let $B$ : $X \rightarrow X$ be continuous, everywhere defined, and dissipative.

Let $L$ be a compact subset of $X$. Let $\left\{f_{n}\right\}$ be a sequence (or more generally, a net) of functions in $\mathcal{L}_{\text {loc }}^{1}(R, X)$ with $\operatorname{Ran}\left(f_{n}\right) \subseteq L$; and let $f_{\infty}$ be another such function.

Then the following two conditions are equivalent:

$$
\begin{gathered}
\lim _{n} u\left(t, f_{n}, a, x\right)=u\left(t, f_{\infty}, a, x\right) \quad \text { for every } x \in X, a \in R, \text { and } t \geqslant a . \\
\lim _{n} \int_{0}^{b} f_{n}(t) d t=\int_{0}^{b} f_{\infty}(t) d t \quad \text { for every } b \in R .
\end{gathered}
$$

Moreover, if (4.1) and (4.2) hold, then the convergence in (4.1) is uniform for $(t, a, x)$ in compact subsets of $R \times R \times X$; and the convergence in (4.2) is uniform for bounded $b$.

REMARKS. 1. For most applications, the implication $(4.2) \Rightarrow(4.1)$ is the useful part of Theorem 1. Generally the functions $f_{n}$ are more directly accessible than the solutions $u\left(\cdot, f_{n}, \cdot, \cdot\right)$; a condition like (4.2) can often be verified directly. The converse implication $(4.1) \Rightarrow(4.2)$ is of interest mainly because it tells us that the useful part cannot be improved.

2. Note that if $\int_{0}^{b}\left\|f_{n}(t)-f_{\infty}(t)\right\| d t \rightarrow 0$ for each $b$, then $u\left(t, f_{n}, a, x\right) \rightarrow$ $u\left(t, f_{\infty}, a, x\right)$ by (2.3). But (4.2) only requires that $\left\|\int_{0}^{b}\left[f_{n}(t)-f_{\infty}(t)\right] d t\right\| \rightarrow 0$. This is a much weaker assumption, especially if the functions $f_{n}$ and $f_{\infty}$ oscillate much. 
Theorem 1 says, roughly, that $u\left(\cdot, f_{\infty}, a, x\right)$ depends only on the average behavior of $f_{\infty}$ over time intervals of positive length, and not on the instantaneous values $f_{\infty}(t)$ at particular times $t$. Similar convergence criteria in other contexts can be found in $[\mathbf{1}, \mathbf{1 5}, \mathbf{2 0}]$, and in papers cited therein.

3. If $A=0$, we can replace the compactness assumption with a boundedness assumption; see Proposition 6.5 of [16] for a more general result. For $A \neq 0$ and $B=0$, the following example shows that the compactness assumption cannot be dropped.

Let $1 \leqslant p<\infty$, and let $X=\varrho^{p}([-\pi, \pi], R)$. Elements of $X$ will be viewed as functions $x(\tau)$ defined for all $\tau \in R$, periodic in $\tau$ with period $2 \pi$. Let $A=\partial / \partial \tau$, with periodic boundary conditions. That is: $(A x)(\tau)=x^{\prime}(\tau)$, with $\operatorname{Dom}(A)=\{x: x$ is absolutely continuous on $[-\pi, \pi], x(-\pi)=x(\pi)$, and $\left.x^{\prime} \in X\right\}$. Then $A$ is linear, densely-defined, and $m$-dissipative. We have $[\exp (t A) x](\tau)=x(t+\tau)$ for all $x \in X$ and $t \geqslant 0$.

For each positive integer $n$, define a continuous function $f_{n}: R \rightarrow X$ by $f_{n}(t)(\tau)=$ $\sin (n t+n \tau)$. Then $\cup_{n} \operatorname{Ran}\left(f_{n}\right)$ is relatively compact in the weak topology of $X$, but not in the norm topology. Let $f_{\infty}=0$; the reader can easily verify condition (4.2).

The differential equation $u_{n}^{\prime}(t)=A\left(u_{n}(t)\right)+f_{n}(t)$ may be rewritten

$$
\frac{\partial u_{n}(t, \tau)}{\partial t}=\frac{\partial u_{n}(t, \tau)}{\partial \tau}+\sin (n t+n \tau) .
$$

For initial value $u_{n}(0, \tau)=0$, the solution is $u_{n}(t, \tau)=t \sin (n t+n \tau)$. That is, $u\left(t, f_{n}, 0,0\right)=t f_{n}(t)$. This does not converge in the norm topology of $X$ when $n \rightarrow \infty$; so (4.1) does not hold.

4. We cannot omit the hypothesis that $A$ is densely defined. For a simple example, let $X$ be the complex Banach space $l^{\infty}$. Let $A\left(x_{1}, x_{2}, x_{3}, \ldots\right)=$ $\left(i x_{1}, 2 i x_{2}, 3 i x_{3}, \ldots\right)$, with $\operatorname{Dom}(A)=\left\{x \in l^{\infty}: \sup _{k} k\left|x_{k}\right|<\infty\right\}$. Then $\overline{\operatorname{Dom}(A)}=$ $c_{0}=$ \{sequences converging to 0$\}$, so $\operatorname{Dom}(A)$ is not dense in $X$.

An easy computation shows that $A$ is $m$-dissipative. Hence $A$ generates a semigroup $\exp (t A)$ on $\overline{\operatorname{Dom}(A)}$. The variation of parameters formula (2.7) is not generally applicable, since the range of $g$ need not lie in $\overline{\operatorname{Dom}(A)}$. But an analogous formula holds separately in each component. The solution of $u^{\prime}(t)=A u(t)+g(t)$ has components $u_{k}(t)=e^{i k t} u_{k}(0)+\int_{0}^{t} e^{i k(t-s)} g_{k}(s) d s(k=1,2,3, \ldots)$.

Now take $B, a, x, f_{\infty}$ all equal to 0 , and $f_{n}(t)=\left(e^{i n t}, e^{i n t}, e^{i n t}, \ldots\right)$. Then an easy computation shows that (4.2) holds but (4.1) does not.

Proof of THE THEOREM. Note that (4.2) is equivalent to

$$
\lim _{n} \int_{a}^{b} f_{n}(t) d t=\int_{a}^{b} f_{\infty}(t) d t \quad \text { for all } a, b \in R \text { with } a<b .
$$

This formulation is more convenient for the proof.

First we show that (4.1) implies (4.3). Let any $\varepsilon>0$ and $a, b \in R$ be given, with $a<b$. Define $\delta=\delta(\varepsilon, L)>0$ as in the lemma in §3. Form a partition: $a=a_{0}<a_{1}$ $<a_{2}<\cdots<a_{m}=b$, where $a_{j}-a_{j-1}<\delta$ for each $j$. Since $m$ is finite, it follows from (3.2) and (4.1) with any $x \in L$ that

$$
\left\|\int_{a_{j-1}}^{a_{j}}\left[f_{n}(s)-f_{\infty}(s)\right] d s\right\|<\left(a_{j}-a_{j-1}\right) \varepsilon \quad(j=1,2,3, \ldots, m)
$$


for all $n$ sufficiently large. Sum over $j$; thus $\left\|\int_{a}^{b}\left[f_{n}(s)-f_{\infty}(s)\right] d s\right\|<(b-a) \varepsilon$. Let $\varepsilon \downarrow 0$; this proves (4.3).

Next assume (4.3) holds; we shall show that the convergence in (4.3) is uniform for all $a, b$ contained in a fixed compact interval [ $p, q]$. Let any $\varepsilon>0$ be given. Let $\alpha=\max \{\|y\|: y \in L\}$. Choose a partition: $p=p_{0}<p_{1}<p_{2}<\cdots<p_{m}=q$, with $\left(p_{j}-p_{j-1}\right) \alpha<\varepsilon$ for each $j$. Since $m$ is finite, it follows from (4.3) that

$$
\max _{0 \leqslant i \leqslant j \leqslant m}\left\|\int_{p_{i}}^{p_{j}}\left[f_{n}(s)-f_{\infty}(s)\right] d s\right\|<\varepsilon
$$

for all $n$ sufficiently large. Consider any such $n$, and any interval $[a, b] \subseteq[p, q]$. Choose $i, j$ so that $p_{i-1} \leqslant a \leqslant p_{i}, p_{j-1} \leqslant b \leqslant p_{j}$, and $i \leqslant j$. Then

$$
\begin{aligned}
\| \int_{a}^{b}\left[f_{n}(s)-\right. & \left.f_{\infty}(s)\right] d s\|\leqslant\| \int_{p_{i}}^{p_{j}}\left[f_{n}(s)-f_{\infty}(s)\right] d s \| \\
& +\int_{a}^{p_{i}}\left\{\left\|f_{n}(s)\right\|+\left\|f_{\infty}(s)\right\|\right\} d s+\int_{b}^{p_{j}}\left\{\left\|f_{n}(s)\right\|+\left\|f_{\infty}(s)\right\|\right\} d s
\end{aligned}
$$

which is less than $5 \varepsilon$. Let $\varepsilon \downarrow 0$; this proves (4.3) holds uniformly for $a, b$ in compact sets.

Finally, assume (4.3) holds uniformly for $a, b$ in compact sets. It suffices to show that (4.1) holds uniformly for $p \leqslant a<t \leqslant q$ and $x \in M$, where $[p, q] \subset R$ and $M \subset X$ are given compact sets. Let any $\varepsilon>0$ be given.

Since $u\left(t, f_{\infty}, a, x\right)$ depends continuously on $(t, a, x)$, the set $M_{1}=\left\{u\left(t, f_{\infty}, a, x\right)\right.$ : $p \leqslant a \leqslant t \leqslant q, x \in M\}$ is compact. Choose $\delta=\delta\left(\varepsilon, L \cup M_{1}\right)>0$ as in the lemma in $\S$. Fix some integer $m>(q-p) / \delta$. By assumption, for all $n$ sufficiently large

$$
\max _{p \leqslant a \leqslant b \leqslant q}\left\|\int_{a}^{b}\left[f_{n}(s)-f_{\infty}(s)\right] d s\right\|<\frac{\varepsilon}{m} .
$$

Now let any $t, a, x$ be given, with $p \leqslant a<t \leqslant q$ and $x \in M$. Since $m \delta>q-p \geqslant$ $t-a$, we can form a partition: $a=a_{0}<a_{1}<a_{2}<\cdots<a_{m}=t$ with $a_{j}-a_{j-1}$ $<\delta$ for each $j$. For each $j$ let $x_{j}=u\left(a_{j}, f_{\infty}, a, x\right)$; then $x_{j} \in M_{1}$. By (2.3), (3.2), and (4.4),

$$
\begin{aligned}
\left\|x_{j+1}-u\left(a_{j+1}, f_{n}, a, x\right)\right\| \leqslant\left\|x_{j+1}-u\left(a_{j+1}, f_{n}, a_{j}, x_{j}\right)\right\| \\
\quad+\left\|u\left(a_{j+1}, f_{n}, a_{j}, x_{j}\right)-u\left(a_{j+1}, f_{n}, a, x\right)\right\| \\
=\left\|u\left(a_{j+1}, f_{\infty}, a_{j}, x_{j}\right)-u\left(a_{j+1}, f_{n}, a_{j}, x_{j}\right)\right\| \\
\quad+\left\|u\left(a_{j+1}, f_{n}, a_{j}, x_{j}\right)-u\left(a_{j+1}, f_{n}, a_{j}, u\left(a_{j}, f_{n}, a, x\right)\right)\right\| \\
\leqslant\left\|\int_{a_{j}}^{a_{j+1}}\left[f_{n}(s)-f_{\infty}(s)\right] d s\right\|+\left(a_{j+1}-a_{j}\right) \varepsilon \\
\quad+\left\|x_{j}-u\left(a_{j}, f_{n}, a, x\right)\right\| \\
\leqslant \varepsilon / m+\left(a_{j+1}-a_{j}\right) \varepsilon+\left\|x_{j}-u\left(a_{j}, f_{n}, a, x\right)\right\| .
\end{aligned}
$$

Summing over $j$, we obtain $\left\|u\left(t, f_{\infty}, a, x\right)-u\left(t, f_{n}, a, x\right)\right\| \leqslant(1+q-p) \varepsilon$. Let $\varepsilon \downarrow 0$; this completes the proof. 
Notation. For any compact interval $[p, q] \subset R$, let $\int([p, q], X)$ be the vector space $\mathcal{L}^{\mathrm{l}}([p, q], X)$ retopologized in either of the following ways. (We present two alternatives, since it is not yet clear which method will yield better generalizations in later research.) (i) $\int([p, q], X)$ is a locally convex topological vector space, with a base of neighborhoods of 0 consisting of sets of the form

$$
\left\{f:\left\|\int_{a_{i}}^{b_{i}} f(t) d t\right\|<\varepsilon_{i}(i=1,2, \ldots, n)\right\}
$$

for finite sets $\left\{a_{1}, a_{2}, \ldots, a_{n}\right\},\left\{b_{1}, b_{2}, \ldots, b_{n}\right\} \subset[p, q]$ and $\left\{\varepsilon_{1}, \varepsilon_{2}, \ldots, \varepsilon_{n}\right\} \subset(0, \infty)$. Then $\int([p, q], X)$ is Hausdorff; see [17]. Alternatively, (ii) $\int([p, q], X)$ is a normed vector space, with norm $\||f|\|=\max _{p \leqslant a \leqslant b \leqslant q}\left\|\int_{a}^{b} f(t) d t\right\|$.

For any compact set $L \subset X$, let $\int([p, q], L)=\left\{f \in \int([p, q], X): \operatorname{Ran}(f) \subseteq L\right\}$. By Theorem 1, topologies (i) and (ii) agree on $\int([p, q], L)$; and the mapping $f \rightarrow u(\cdot, f, p, x)$ is continuous from $\int([p, q], L)$ into $\mathcal{C}([p, q], L)$ for any fixed $x \in X$. If $L$ is convex, then $\int([p, q], L)$ is obviously convex; it is also compact, by Lemma 2 of [17]. Combining these observations with (2.3), we obtain

Corollary. Let $A, B$ be as above. Let $K, L$ be compact subsets of $X$, with $L$ convex. Let $0<T<\infty$. Then $\left\{u(t, f, 0, x): t \in[0, T], f \in \mathcal{L}^{1}([0, T], X), \operatorname{Ran}(f) \subseteq\right.$ $L, x \in K\}$ is compact.

\section{An existence theorem.}

Theorem 2. Let $A$ be a linear, densely-defined, $m$-dissipative operator in $X$. Let $B$ : $X \rightarrow X$ be continuous and dissipative. Let $C:[0, \infty) \times X \rightarrow X$ be compact; i.e., assume $C$ is jointly continuous and maps bounded sets to relatively compact sets. Let $x \in X$.

Then for some $T=T(x)>0$ there exists a solution of $u^{\prime}(t)=(A+B)(u(t))$ $+C(t, u(t))(0 \leqslant t<T) ; u(0)=x$. That is, $u$ is an integral solution of $u^{\prime}(t)=$ $(A+B)(u(t))+f(t)$ on $[0, T)$, with $u(0)=x$ and $f(t)=C(t, u(t))$.

Moreover, suppose $T$ is chosen maximally; i.e., the solution $u$ cannot be continued further. If $T<\infty$ then $\{u(t): 0 \leqslant t<T\}$ is unbounded and $\{C(t, u(t)): 0 \leqslant t<T\}$ is not relatively compact. In particular, if $\operatorname{Ran}(C)$ is relatively compact then $T$ must be $\infty$.

Remark. The hypothesis that $C$ is jointly continuous can be replaced with the weaker assumption that $C$ satisfies Carathéodory conditions (see $[16,17])$. Also, $B$ can easily be made time-dependent. For brevity we omit the details.

ProOF OF THE THEOREM. First we show local existence; i.e., we prove that for each $x \in X$ and $a \geqslant 0$ there exists a solution of $u^{\prime}(t)=(A+B)(u(t))+C(t, u(t))$ on $[a, a+\tau]$ with $u(a)=x$, for some $\tau=\tau(a, x)>0$.

Let $K$ be the closed convex hull of $\{C(t, y): t \in[a, a+1], y \in X,\|x-y\| \leqslant 1\}$. Then $K$ is compact. Define $\delta=\delta(1, K \cup\{x\})>0$ as in the lemma in $\S 3$. Let $\tau=\min \{\delta, 1\}$. Define $\int([a, a+\tau], X)$ and $\int([a, a+\tau], K)$ as in $\S 4$.

The mapping

$$
f \mapsto u(\cdot, f, a, x)
$$

is continuous from $\int([a, a+\tau], K)$ into $\mathcal{C}([a, a+\tau], X)$. By (3.1),

$$
\|u(t, f, a, x)-x\|<1
$$


for $f \in \int([a, a+\tau], K)$ and $t \in[a, a+\tau]$; hence $C(t, u(t, f, a, x)) \in K$. The mapping $u \mapsto C(\cdot, u(\cdot))$ is continuous from $\Theta([a, a+\tau], X)$ into $\int([a, a+\tau], X)$. Hence $f \mapsto C(\cdot, u(\cdot, f, a, x))$ is a continuous self-mapping of the compact convex set $\int([a, a+\tau], K)$. By the Schauder-Tychonoff Theorem [10, Theorem V.10.5], this mapping has at least one fixed point $f_{0}$. Then $u\left(\cdot, f_{0}, a, x\right)$ is the required solution. This completes the proof of local existence.

Now fix some particular $x_{0} \in X$, and let $u_{0}^{\prime}(t)=(A+B)\left(u_{0}(t)\right)+C\left(t, u_{0}(t)\right)$ with initial value $u_{0}(0)=x_{0}$. By Zorn's Lemma, $u_{0}$ can be extended to a maximal interval $[0, T)$. (See [13, Lemma VI.1.2] for a similar argument.)

Suppose that $T<\infty$ but $L_{1} \equiv\left\{C\left(t, u_{0}(t)\right): 0 \leqslant t<T\right\}$ is relatively compact. We shall obtain a contradiction.

Indeed, let $L$ be the closed convex hull of $L_{1}$; then $L$ is compact and convex. By the corollary at the end of $\S 4, M \equiv\left\{u\left(t, f, 0, x_{0}\right): 0 \leqslant t \leqslant T, f \in \int([0, T], L)\right\}$ is compact. Note that $M$ contains the range of $u_{0}$.

For any $\varepsilon>0$, define $\delta=\delta(\varepsilon, L \cup M)>0$ as in the lemma of $\S 3$. By (3.1), $\left\|u_{0}(t)-u_{0}\left(t^{\prime}\right)\right\|<\varepsilon$ whenever $\max \{0, T-\delta\} \leqslant t \leqslant t^{\prime}<T$. Hence $u_{0}(t)$ converges to some limit as $t \uparrow T$; denote the limit by $u_{0}(T)$. Take limits in (2.2) or (2.8); it follows that $u_{0}$ is an integral solution of $u_{0}^{\prime}(t)=(A+B)\left(u_{0}(t)\right)+C\left(t, u_{0}(t)\right)$ on the closed interval $[0, T]$.

Use the local existence result to obtain a solution on $[T, T+\tau]$ for some $\tau>0$. Combine the solutions on $[0, T]$ and $[T, T+\tau]$ to obtain a solution on $[0, T+\tau]$. This contradicts the maximality of $T$.

Thus $T<\infty$ implies $L_{1}$ is not relatively compact. Then $\{u(t): 0 \leqslant t<T\}$ is unbounded, since $C$ is compact. This completes the proof of the theorem.

\section{REFERENCES}

1. Z. Artstein, Continuous dependence of solutions of operator equations. I, Trans. Amer. Math. Soc. 231 (1977), 143-166.

2. V. Barbu, Continuous perturbations of nonlinear m-accretive operators in Banach spaces, Boll. Un. Mat. Ital. 6 (1972), 270-278.

3. Nonlinear semigroups and differential equations in Banach spaces, Noordhoff, Leyden, 1976.

4. A. Belleni-Morante, Applied semigroups and evolution equations, Oxford Univ. Press, Oxford, 1979.

5. P. Bénilan, Equations d'évolution dans un espace de Banach quelconque et applications, Thèse, Orsay, 1972.

6. P. Bénilan, M. G. Crandall, and A. Pazy (to appear).

7. M. G. Crandall and L. C. Evans, On the relation of the operator $\partial / \partial s+\partial / \partial \tau$ to evolution governed by accretive operators, Israel J. Math. 21 (1975), 261-278.

8. M. G. Crandall and T. M. Liggett, Generation of semi-groups of nonlinear transformations on general Banach spaces, Amer. J. Math. 93 (1971), 265-298.

9. M. G. Crandall and A. Pazy, An approximation of integrable functions by step functions with an application, Proc. Amer. Math. Soc. 76 (1979), 74-80.

10. N. Dunford and J. T. Schwartz, Linear operators. I, Wiley, New York, 1957.

11. L. C. Evans, Application of nonlinear semigroup theory to certain partial differential equations, in Nonlinear Evolution Equations (M. G. Crandall, ed.), Academic Press, New York, 1978.

12. J. A. Goldstein, Semigroups of operators and applications, Addison-Wesley, Reading, Mass. (to appear).

13. R. H. Martin, Jr., Nonlinear operators and differential equations in Banach spaces, Wiley, New York, 1976.

14. M. Pierre, Perturbations localement Lipschitziennes et continues d'operateurs m-accretifs, Proc. Amer. Math. Soc. 58 (1976), 124-128. 
15. E. Schechter, One-sided continuous dependence of maximal solutions, J. Differential Equations 39 (1981), 413-425.

16. Existence and limits of Carathéodory-Martin evolutions, J. Nonlinear Anal. Theory Methods Appl. 5 (1981), 897-930.

17. Evolution generated by continuous dissipative plus compact operators, Bull. London Math. Soc. 13 (1981), 303-308.

18. Interpolation of nonlinear partial differential operators and generation of differentiable evolutions, J. Differential Equations (to appear).

19. P. Volkmann, Ein Existenzsatz für gewöhnliche Differentialgleichungen in Banachräumen, Proc. Amer. Math. Soc. 80 (1980), 297-300.

20. Z. Vorel, Continuous dependence on parameters, J. Nonlinear Anal. Theory Methods Appl. 5 (1981), $373-380$.

Department of Mathematics, Vanderbilt University, NashVille, TenNessee 37235 